\title{
PHYSICAL THERAPY OF BEACH SOCCER ATHLETES SUFFERING OF METATARSALGIA
}

\author{
Polina Todorova, Nikolay Popov \\ National Sports Academy "Vassil Levski", Sofia, Bulgaria
}

\begin{abstract}
The research paper presents detailed physical therapy program for recuperation of muscle function and motor control in the ankle and foot zone. It is applicable to subacute or chronic recovery after different disorders in the respective human body area and after the end of effective arthrokinematic healing procedures.

Ten individual cases of beach football players have been observed during the research, itself. The balance of the body during stance and gait requires a delicate combination of mobility and stability. Reference terms and muscle tone recovery methods, relaxation of the weak muscle as techniques have been used. Exercises in a closed kinetic chain, in respect to body weight and joint-mobilization, corresponding to relevant techniques, and elastic resistance exercises have been focused.

Statistically, application of highly-profiled therapeutic exercises have resulted into a significant decrease of pain levels and increase of pain-free range of motion. The aim has been to use soft tissue mobilizations, natural proprioceptive facilitation of muscle function and to recover the complex functional abilities of ankle and foot locomotion. Respective biometrical and functional features of the ankle and foot as some of the most loafed areas of muscle skeletal system have been taken into consideration.

An algorithm for application of the most effective physical therapy methods in that kind of foot traumas has been investigated and described.
\end{abstract}

Key words: foot, ankle, physical therapy, joint-tissue mobilization, muscle function

\section{INTRODUCTION}

Ankle and foot complex is a structure providing stability and mobility of the distal portion of the lower lib. When walking, this segment works as a flexible lever, and when standing - as a rigid structure that supports the entire body. The kinematics of the ankle/foot complex successfully ensures the adaptation to terrain roughness (Popov, Popova, Grueva, 2013).

In addition to the well-known lower limb injuries in contact sports, caused by falls and various accidents, there are also other equally complicated and painful foot dysfunctions observed in beach soccer. They are related to the terrain specificity, complex techniques used in the game as well as injuries related to feet biomechanical positions on an unstable support surface (Giza, Fuller, Junge, 2003). Moving on uneven sandy terrain is a great test for each player's musculoskeletal system. The effective and atraumatic practicing of beach soccer is a combination of well-developed balance and movement control on the pitch and perfect ball possession. Various combinations of speed, strength and explosiveness are widely used in this type of sport (Schirinzi, Amarelle, Barrabe, Montserrat, Close, Gürlet, 2015). Constant biomechanical changes in the movement are consistent with maximum impact and rapidly changing playing configurations and conditions; this, in turn, picks up injury risk (Cormick, Anderson,2010).

The most common injuries, encountered during the beach soccer game, are those in the foot and toe zones. Despite the widespread injuries that can may be experienced by all footballers on all types of terrain, here we can observe specific clinical syndromes that cover metatarsophalangeal and interphalangeal joints. Hallux is the most common trauma. The metatarsophalangeal joint synovitis, or metatarsalgia, is a general term for pain in the metatarsal area. This inflammatory disease often covers the first metatarsophalangeal join (Durham, 2017).

According to Medical Commission, Union of European Football Associations, research, the beach soccer foot traumas are rating at first position. The increasing complaints and strain in the foot area occurring in soccer players determines the current interest to developing and introducing methodologies that are easy to be implemented by the athletes, themselves, and, at the same time, effectively influencing pain onset patokinesiological mechanisms, while significantly improving their life quality (Valderrabano, Barg, Paul, 2014). 
Around $80 \%$ of human population suffers of some foot and ankle complaints. Most of these pathologies can be avoided. Foot lesions affect the gait mechanics and increase, reflexively, the stress in other lower limb joints. This may result in their future injury. Some of the most common traumas that compromise proper function and patient's overall body support are bone spurs (exostosis), Morton's neuroma, plantar fasciitis, fractures of the sesamoid bones, Pesplanus, Halluxvalgus, Halluxrigidus, Turftoe, Tarsaltunnelsyndrome, etc. (Rampinini, Castagna, Martino, Fiorini, Wisloff, 2015).

However, individual segments' structure and functioning are very important for the successful identification and overcoming the advancing pathokinesiological changes (Popov, Popova, Grueva, 2012). Foot mobility determines its shock absorption properties and its ability to adapt to rough terrains. Foot, itself, is a multi-segmental structure and its various segments play various roles in its overall function (Popov, 2009).

With the advancement of studies in the field of foot/ankle complex and associated pathological conditions, it is becoming easier the condition to be diagnosed and managed at an early stage.

Proper identification of the occurred functional and structural damage and dysfunctions is of crucial importance, while determining the management approach. In many cases, it is possible to precisely determine the anatomic structure affected and its healing stage. This has a great impact over the clinical treatment. In case of milder sprains and strains, conservative treatment, involving rest and RICE method (rest, ice, compression and elevation), is recommended. Next is coming foot pain physiotherapy program. It is also successfully applied to reduce pain caused by injuries in their acute and chronic stage. Corticosteroid should not be injected because they may increase the risk of further damage and mask the symptoms (Kakwani, et al., 2018). Indications to surgery include major capsular avulsions with instability of the joint, unstable fractures of the sesamoid (Giza, Fuller, Junge, 2003).

The main tasks, needed to design proper patient's recreation activities for an effective recuperation of limb function, depend on his/her individual status and pathological findings. Non-surgical management involves counteracting inflammatory processes, joint mobility improvement, limb strength and functionality recuperation. As early as patient's first complaint opens, better kinesitherapeutic treatment. Therapist's eye is focused on improving the biomechanical function of the foot and regaining neuromuscular control (Wilkersor, Fischer, 2017)

\section{METHODS}

Our survey included a group of 10 beach soccer athletes, all were males. All had been experiencing foot pain for more than half an year. The patients were examined and individually trained in the kinesitherapeutic complex. Therapeutic course was conducted within 40 days. The treatment techniques selected were matched with the players training process and respectively included in an adequate manner. Players performed the exercises independently according to a predetermined program. The exercise dosage was fixed in three sets of ten repetitions performed in a circular workout. Physiotherapeutic approach methodology was approved, respectively, by a profiled medical doctor. Collaboration with the patient was of paramount importance as well as taking into account his expectations and wishes for the activities of his daily life. Training of patients to perform their exercises and mobilization techniques unattended is of significance to maintain the results over time (Figures $1,7)$.

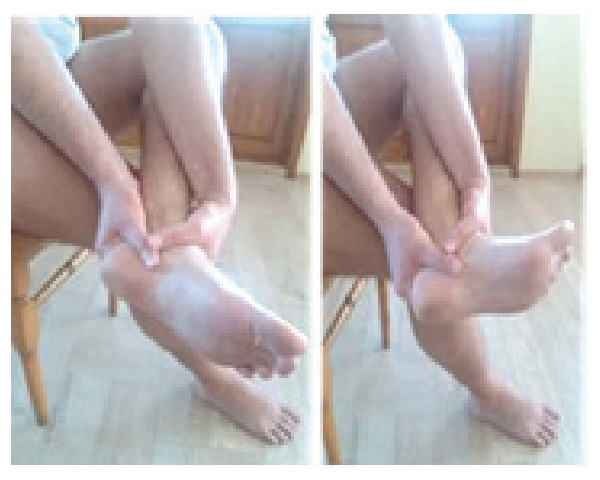

Figure 1. Subtalar joint distraction 
The kinesitherapeutic approach depends on the clinical counts and symptoms. The basic guidelines for acute, subacute and chronic joint problems were observed. In addition, the application of the following physiotherapeutic instrumentarium is appropriate to be applied:

- manual physical therapy techniques (Figures 1, $6,7)$;

- soft tissue mobility maintaining techniques (Figures 3, 9, 10);

- joint mobility maintaining techniques (Figures $1,6,9)$;

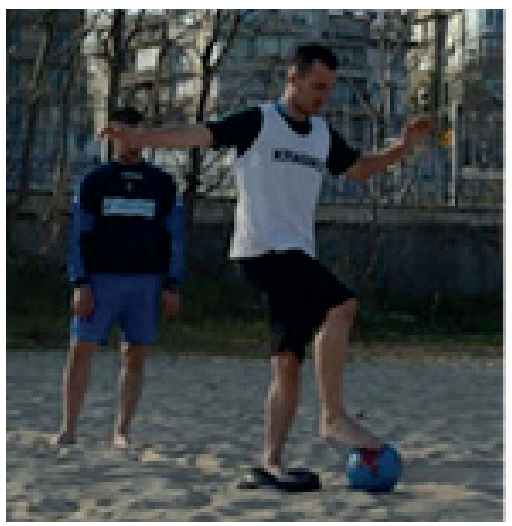

Figure 2. Keeping balance on the board
- muscle function restoring techniques (Figures $3,4,5,8)$;

- neuromuscular control improving techniques (Figures 2, 5)

Distraction and oscillation techniques with active movements contributing to pain suppression were used. Appropriate active and multi-angular exercises were selected on the basic guidelines for acute, subacute and chronic joint problems (Popov, Popova, Grueva, 2012), increasing soft tissue mobility and elasticity (Figure 3), m. Triceps surae stretch (Figure 4), etc.

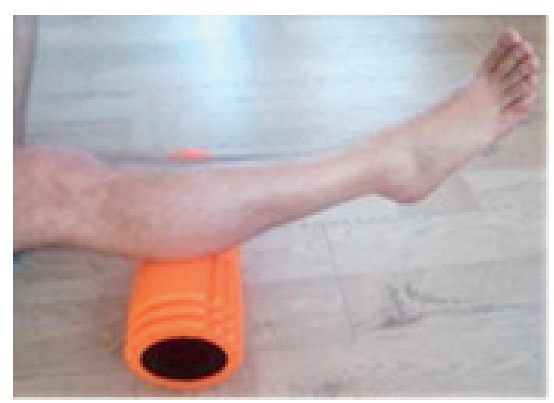

Figure 3. Processing of m.triceps surae

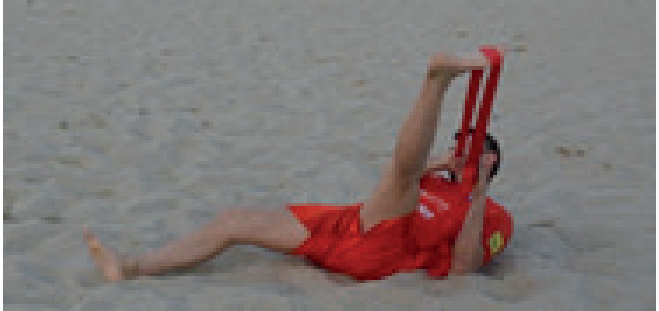

Figure 4. Stretching for ischiocrurale muscle, m.triceps surae

Complex motor skills recovery and restoration of Having targeted kinesitherapy to overcome foot proprioception are an integral part of the correct selection of kinesitherapeutic program. Means in foot injuries, C. Nery, MD, an international member of the American Orthopedic Foot and Ankle Society (AOFAS), recommends kinesitherapy techniques to improve joint mobility and exercises to strengthen and improve coordination skills (Nery, traumatic injuries would allow for further prevention of more serious injuries to the ankle, knee and hip joints (Valderrabano, Barg, Paul, 2014).

The complex includes rising onto the toes with the weight of the body on an inclined plane to improve the strength, endurance and proper synergy of the underlying muscles (Figure 5).

Raduan, Baumfeld, 2016).
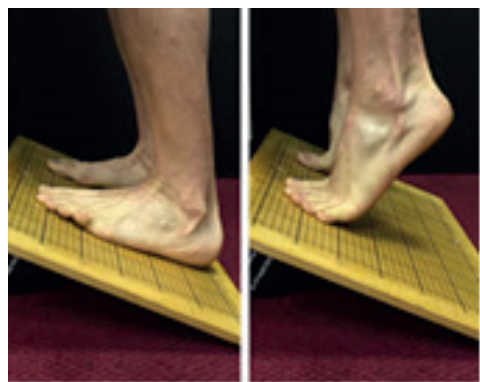

Figure 5. Lifting of fingers and slow relaxation until complete inclination of inclined surface. 
Mobilization techniques were tailored to the individual training of the football players and aimed

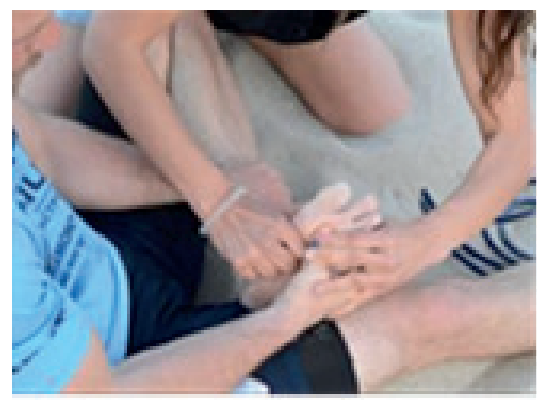

Figure 6. Training for self-fulfillment medial side to improve joint mobility and to maintain positive effect over time (Figures 6, 7).

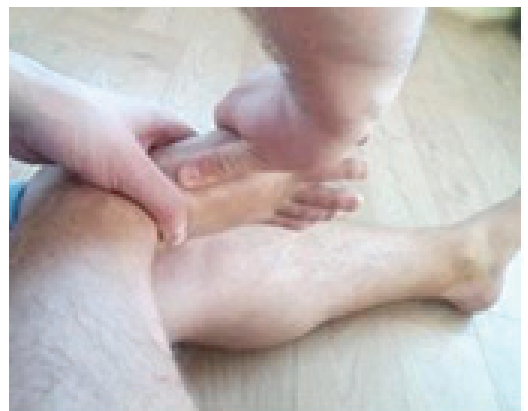

Figure 7. Mobilization of the tarsal bones on the of mobilization Techniques - mobilization of the tarsal bones.

The complex included resistance band exercises for durance (Figure 8).

better strength and muscle coordination and en-
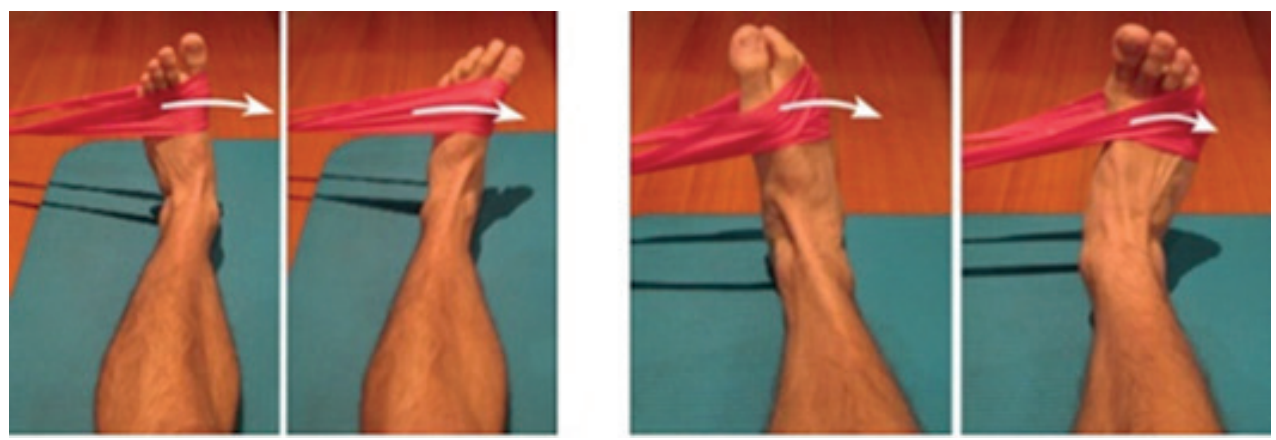

Figure 8. Performance of inversion and eversion against elastic resistance

Balance and one leg stand exercises with overcoming body weight were also involved. Foot was positioned in the area of the metatarsophalangeal

joints to make the exercise more complex and to improve muscle synergy in the foot region (Figure 9).
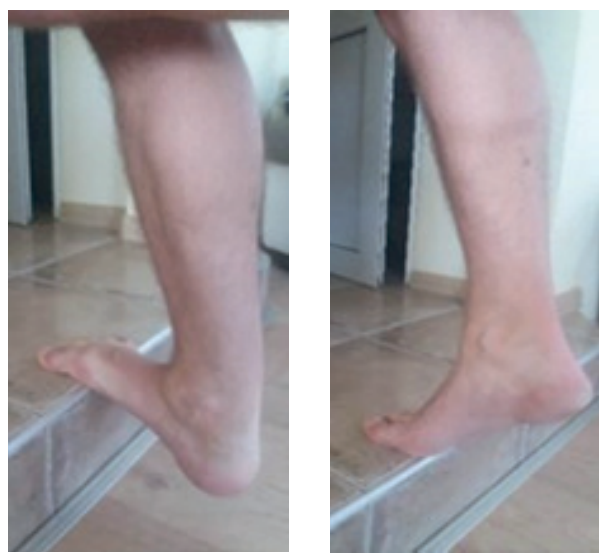

Figure 9. The foot has reached the area of the tarsometaphalangeal joints.

The patient is lifted to the chest then lowered slowly.

To alleviate pain and prevent complications, the mobility (Durham, 2017).

American Association of Orthopaedic Surgeons The complex included solid ball exercises to treat (AAOS) recommends structured physical therapy plantar fascia. The patient is in standing position. usage involving exercises for plantar fascia. Fol- A solid ball is used, on which the patient applies lowing injuries and/or surgeries, the AAOS recom- pressure with his body weight on the plantar surmends exercises improving ankle/foot complex face of his foot. The foot is rolled over the ball as the 
exercise is carried under control with a continuous pressure on a stable, solid support. This exercise aims to alleviate the pain in the foot/ankle complex and improve the function of the underlying plantar structures (Figure 10).

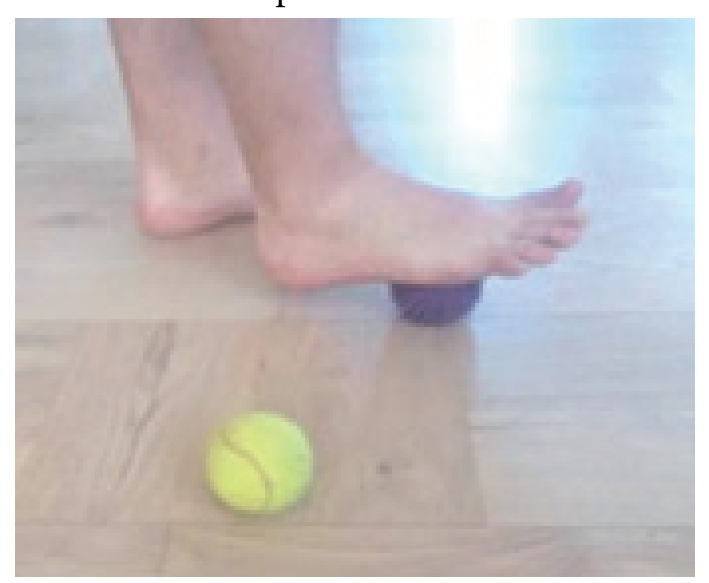

Figure 10. Solid plantar fascia treatment

\section{RESULTS}

In our research process the tests and measurements were made at the beginning, in the middle and at the end of the kinesitherapy treatment. So, the data obtained enabled us to check the selected tests' reliability. The exercises selected and systematized in the kinesitherapy program had beneficial impact on the leading pain and discomfort symptom while playing and walking. The complex of specialized kinesitherapy exercises had a positive effect on the players' functional capabilities; their neuromuscular control was significantly improved;

The complaints of discomfort and pain localized in the foot zone were significantly reduced. The findings of our study demonstrated statistically significant improvement in pain-free (Range Of Motion, ROM) (Figure 11). The effect of the exercise therapy in reducing pain is mostly related with improved treatments, such as manual-mobilization techniques, stretching and modalities.

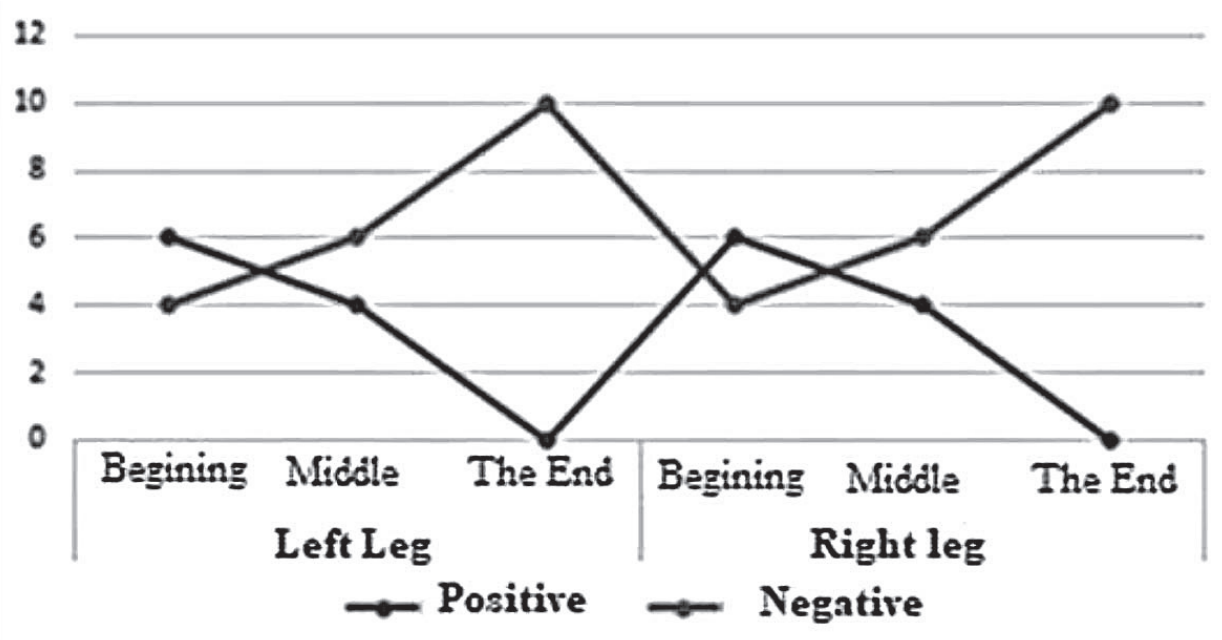

Figure 11. Shows the results of the plantar and dorsi-flexion angle measurements.

The results of the test (Windlass test) show a significant improvement among the players after the therapeutic program (Figure 12). At the beginning of the study, 9 out of 10 patients had a positive result (left foot). At the end of the study, 10 sports were negative. 


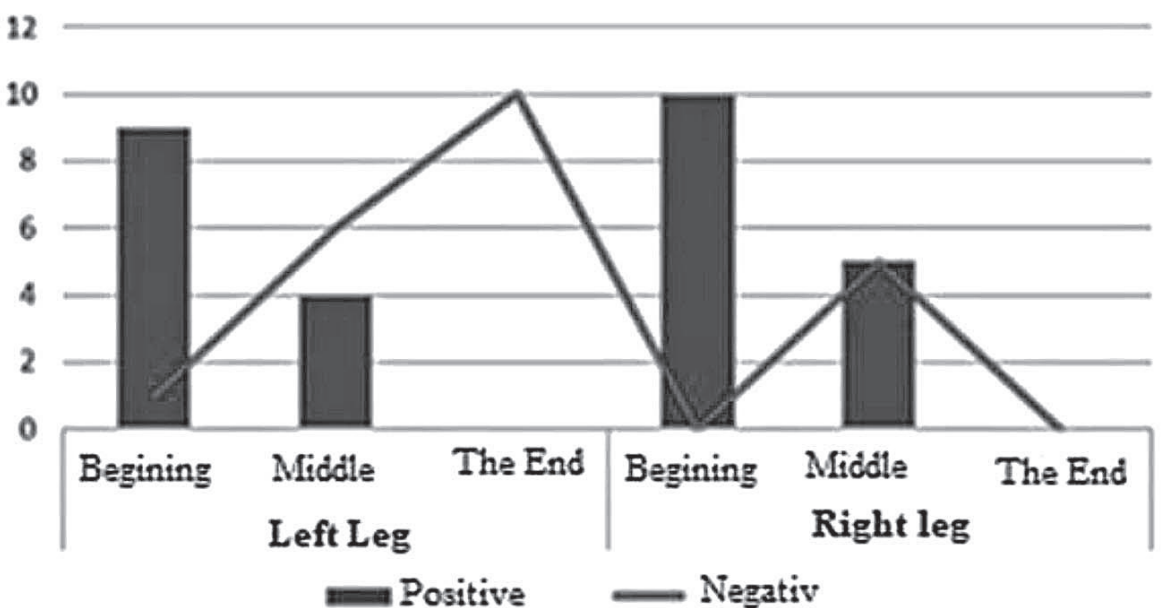

Figure 12. Shows the results of Windlass test.

The data obtained from the pain intensity examination showed that at the beginning of the treatment the pain level was average 6 (VAS) and at the end VAS score decreased to 2 .

The exercise therapy effect on pain reducing is principally related to specific treatments, triggering far-reaching improvement, and their combination with tools such as mobilization techniques, stretching and modalities.

\section{CONCLUSION}

The data from the survey shown that Complex motor skills recovery and restoration of proprioception are an integral part of the correct selection of kinesitherapeutic program. Physiotherapy for athletes with metatarsalgia is a complex process that requires detailed and comprehensive evaluation and identification of causes of foot pain.

\section{REFERENCES}

Durham, BA. (2012). Metatarsalgia Treatment \& Management.

Rampinini, E., Castagna, C., Martino, F., Fiorini, S., Wisloff, U. (2008). Effect of plyometric training on sand versus grass on muscle soreness and jumping and sprinting ability in soccer players;

Schirinzi, A., Amarelle, R., Barrabe, C., Montserrat, D., Close, M., Gürlet, T. (2015). FIFA Education \& Technical. Giza, E., Fuller, C., Junge, A., et al. (2003). Mechanisms of foot and ankle injuries in soccer. Am J Sports Med;
Hennessy, MS., Saxby, TS. (2001). Traumatic "mallet toe" of the hallux: a case report. Foot Ankle Int.

McCormick, JJ., Anderson, RB. (2010). Rehabilitation following turf toe injury and plantar plate repair. Clin Sports Med.

Kakwani, R., Haque, S., Chadwick, C., Davies, M., Blundell, C. (2018). Metaphyseal Osteotomy (DMMO) for Lesser Toe Metatarsalgia. Foot \& Ankle International;

Nery, C., Baumfeld, R. (2016). Foot and Ankle Injuries in Professional Soccer Players. Diagnosis, Treatment, and Expectations, Foot Ankle Clin.

Popov, N. (2009). Kinesiology and pathokinesiology of musculoskeletal system, NSA PRESS, Sofia.

Popov, N., Popova, D., Grueva, T. (2013). Physiotherapy for musculoskeletal dysfunction of the lower extremities, NSA PRESS, Sofia.

Popov, N., Popova, D., Grueva, T. (2012). Functional research and analysis in musculoskeletal physiotherapy, NSA PRESS, Sofia.

Valderrabano, V., Barg, A., Paul, J., et al. (2014). Foot injuries in professional soccer players. Sports Orthopaedics and Traumatology.

Wilkersor, R., Fischer, J. (2017). The prevention of injury.

\section{Corresponding autor: \\ Polina Todorova \\ PhD-student,}

Department of Theory and methodology of kinesitherapy National Sports Academy "Vassil Levski”, Sofia E-mail: politodorova93@gmail.com 